\title{
The nature of electron correlation in a dissociating bond
}

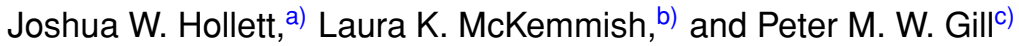 \\ Research School of Chemistry, Australian National University, Canberra, \\ Australian Capital Territory 0200, Australia
}

(Received 14 April 2011; accepted 23 May 2011; published online 10 June 2011)

\begin{abstract}
We have constructed the unrestricted Hartree-Fock (UHF), restricted Hartree-Fock (RHF), and full configuration interaction (FCI) position and momentum intracules and holes for $\mathrm{H} \cdots \mathrm{H}$ at bond lengths $R$ from 1 to 10 bohrs. We trace the recently discovered inversion of the UHF position hole at intermediate $R$ to over-localization of the spin-orbitals, and support this by a correlation energy component analysis. The RHF and UHF momentum holes are found to be more complicated; however their features are explained through decomposition of electron correlation effects. The UHF momentum hole is also found to invert and exhibits interesting behavior at large $R$. The RHF (but not UHF) and FCI momentum intracules exhibit Young-type interference patterns related to recent double photoionization experiments. Our analyses yield the most comprehensive picture to date of the behavior of the electrons during homolytic bond fission. (C) 2011 American Institute of Physics. [doi:10.1063/1.3599937]
\end{abstract}

\section{INTRODUCTION}

New approaches to the electron correlation problem, including natural orbital functional theory, ${ }^{1-3}$ intracule functional theory, ${ }^{4-9}$ and new density functionals, ${ }^{10}$ continue to emerge but their effective development depends critically on improved understanding in simple model systems. The $\mathrm{H} \cdots \mathrm{H}$ molecule, for example, is the prototype of homolytic bond fission and yet, even in this simple case, a deep understanding of electron correlation effects remains elusive. ${ }^{11}$

The correlation energy ${ }^{12}$ is the difference,

$$
E_{\mathrm{c}}=E_{\text {exact }}-E_{\mathrm{HF}},
$$

between the exact non-relativistic energy and the HartreeFock (HF) energy. In the Löwdin definition, ${ }^{13}$ the HF energy is calculated in a spin-restricted Hartree-Fock (RHF) framework; in the Pople definition, ${ }^{14}$ a spin-unrestricted HartreeFock (UHF) framework is assumed. Electron correlation effects are sometimes very different in these frameworks.

The behavior of the correlation energy in the groundstate $\mathrm{H} \cdots \mathrm{H}$ system as the bond length $R$ increases depends strongly on the chosen definition. Whereas the Löwdin correlation energy rises toward a value exceeding $5 \mathrm{eV}$ (because RHF cannot describe homolytic bond fission), the Pople correlation energy reaches a maximum near $R=2.4$ bohrs and then decreases to zero as the bond is lengthened further. ${ }^{11}$

Two-electron probability distribution functions, or intracules, are useful tools for examining correlation effects. The best known are the position intracule ${ }^{15}$

$$
P(\mathbf{u})=\frac{N(N-1)}{2}\left\langle\Psi\left|\delta\left(\mathbf{r}_{1}-\mathbf{r}_{2}-\mathbf{u}\right)\right| \Psi\right\rangle,
$$

\footnotetext{
a) Electronic mail: jhollett@rsc.anu.edu.au.

b) Electronic mail: 1mckemmish@ rsc.anu.edu.au.

c)Electronic mail: peter.gill@anu.edu.au.
}

and the momentum intracule ${ }^{16}$

$$
M(\mathbf{v})=\frac{N(N-1)}{2}\left\langle\Phi\left|\delta\left(\mathbf{p}_{1}-\mathbf{p}_{2}-\mathbf{v}\right)\right| \Phi\right\rangle,
$$

where $\Psi\left(\mathbf{r}_{1}, \ldots, \mathbf{r}_{N}\right)$ and $\Phi\left(\mathbf{p}_{1}, \ldots, \mathbf{p}_{N}\right)$ are the positionand momentum-space wavefunctions of the system, respectively. The spherical averages of these functions are the radial intracules

$$
\begin{gathered}
P(u)=\int P(\mathbf{u}) d \Omega_{\mathbf{u}}, \\
M(v)=\int M(\mathbf{v}) d \Omega_{\mathbf{v}},
\end{gathered}
$$

which are the probability densities of the relative distance $u=|\mathbf{u}|$ and momentum $v=|\mathbf{v}|$ of pairs of electrons.

Correlation effects can be seen in the intracule holes

$$
\begin{gathered}
\Delta P(u)=P_{\text {exact }}(u)-P_{\mathrm{HF}}(u), \\
\Delta M(v)=M_{\text {exact }}(v)-M_{\mathrm{HF}}(v),
\end{gathered}
$$

arising from the differences between intracules from correlated and HF wavefunctions.

There have been many previous investigations ${ }^{17-23}$ of the position and momentum intracules of $\mathrm{H}_{2}$ and these involved both the anisotropic and spherically averaged forms of the intracules. Most of these studies focused on $\mathrm{H}_{2}$ at its equilibrium bond length but Boyd et al. ${ }^{19}$ investigated $\Delta P_{\mathrm{RHF}}(u)$ and Per et al. ${ }^{23}$ computed $\Delta P_{\mathrm{UHF}}(u)$ as functions of $R$. The features of $\Delta P_{\mathrm{RHF}}(u)$ are straightforward and reflect the inability of RHF to describe homolytic fission. Per et al. observed that $\Delta P_{\mathrm{UHF}}(u)$ is more complicated but they did not discuss the origin of this complexity. $\Delta M_{\mathrm{RHF}}(v)$ and $\Delta M_{\mathrm{UHF}}(v)$ have not previously been studied as functions of $R$.

Therefore, to improve our understanding of this fundamental system, we have determined the cause of the unexpected behavior of $\Delta P_{\mathrm{UHF}}(u)$ and we have constructed both $\Delta M_{\mathrm{RHF}}(v)$ and $\Delta M_{\mathrm{UHF}}(v)$. In Sec. II, we discuss $\Delta P_{\mathrm{RHF}}(u)$ 


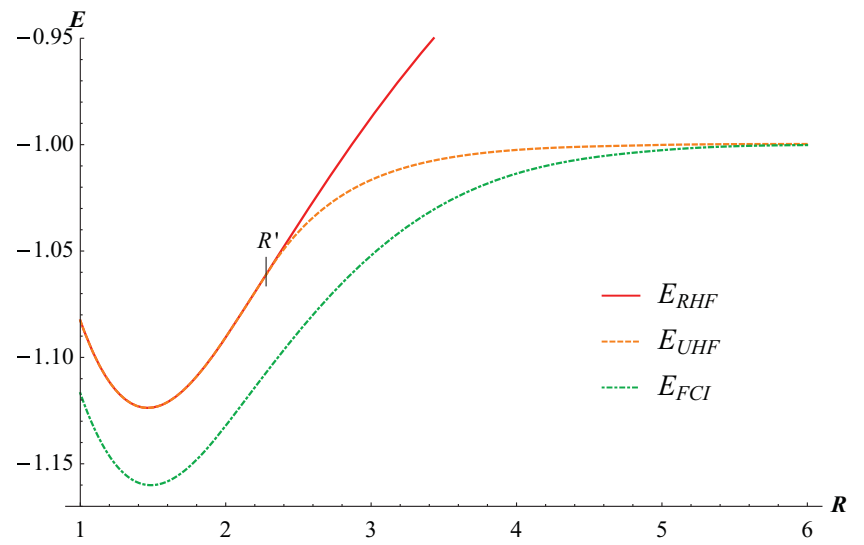

FIG. 1. RHF, UHF, and FCI energies of $\mathrm{H}_{2}$ for $1 \leq R \leq 6$.

and $\Delta P_{\mathrm{UHF}}(u)$ for $1 \leq R \leq 10$, decomposing the correlation energy and explaining the unexpected shape of $\Delta P_{\mathrm{UHF}}(u)$ at large $R$. In Sec. III, we discuss the corresponding behavior of the momentum holes and investigate this using a simple model. Atomic units are used throughout.

\section{POSITION HOLES}

A recent study ${ }^{8}$ found that the gross features of intracules are largely insensitive to basis set improvements and, accordingly, we adopted the $6-311 \mathrm{G}(d, p)$ basis throughout the present work. In this basis, the RHF wavefunction develops a triplet instability at the bond length $R^{\prime}=2.285$ (Fig. 1), which is similar to the infinite-basis value $\left(R^{\prime}=2.296\right)$ found by Rassolov et al. ${ }^{11}$

To construct position holes, it is necessary to compute the RHF, UHF, and full configuration interaction (FCI) intracules. These were constructed ${ }^{9,24}$ from wavefunctions computed using the GAMESS software package. ${ }^{25}$

In the RHF model, the electrons share a $\sigma_{g}$ orbital and the probability of small $u$ values is therefore large. However, if correlation is introduced, the electrons are able to settle at opposite ends of the molecule and $r_{12} \approx R$. Thus, as Boyd discovered, ${ }^{19} \Delta P_{\mathrm{RHF}}(u)$ has a minimum at small $u$ and a maximum near $u=R$ (Fig. 2). Recently, Per et al. discovered that the hole also possesses a shallow secondary minimum at large $u$ values $(u \gtrsim 5)$. An analogous secondary minimum has also

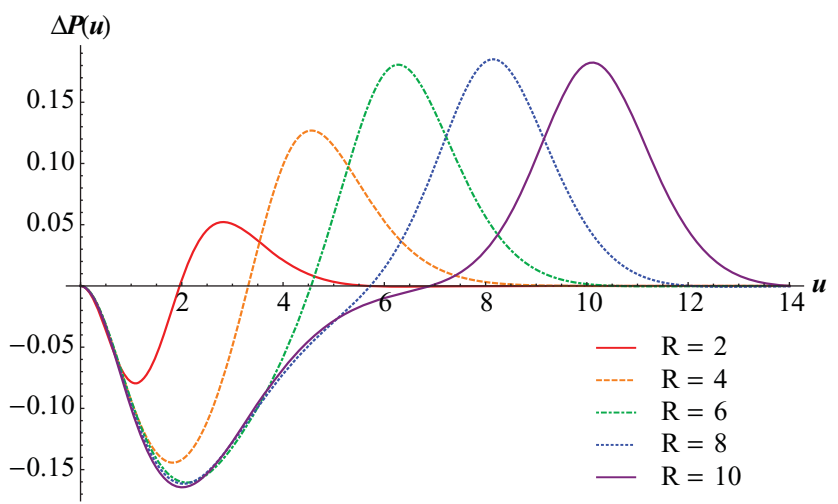

FIG. 2. RHF position holes for various $R$.



FIG. 3. UHF position holes for various $R$.

been observed for the $\mathrm{He}$ atom ${ }^{26}$ and electrons on concentric spheres. $^{27}$

In the UHF model, things are more complicated. For $R<R^{\prime}$, the UHF and RHF holes are of course identical but, as $R$ grows beyond $R^{\prime}$, the UHF treatment allows each electron to localize around a nucleus and the holes, therefore, become completely different (Fig. 3). The primary minimum diminishes and the secondary minimum deepens until, near $R=2.9$, the latter becomes more significant than the former. If the bond is extended to $R=3.5$, the primary minimum disappears altogether, leading to a "hole inversion" that was first reported by Per et al. This leads to the unexpected conclusion that, for $R>3.5$, the introduction of electron correlation (in the UHF framework) serves to bring the electrons closer together. ${ }^{26}$ As the bond is stretched still further, the inverted hole weakens and eventually vanishes.

The hole inversion is the signature of a qualitative change in the physics of electron correlation and we can show this by analyzing the kinetic $T_{\mathrm{c}}$, nuclear-electron $V_{\mathrm{c}}$, and electronelectron $J_{\mathrm{c}}$ components of $E_{\mathrm{c}}$ (Fig. 4).

For $R<2.6$, where the hole is normal, we find that $T_{\mathrm{c}}$ is positive, but $V_{\mathrm{c}}$ and $J_{\mathrm{c}}$ are negative, implying that correlation stabilizes the system by improving the Coulombic interactions at the expense of increasing the kinetic energy. This picture is consistent with the conventional wisdom that correlation allows electrons to avoid one another but that, to do so, they must move more quickly.

For $R>2.9$, where the hole is inverted, we find that $T_{\mathrm{c}}$ is negative, but $V_{\mathrm{c}}$ and $J_{\mathrm{c}}$ are positive, implying that

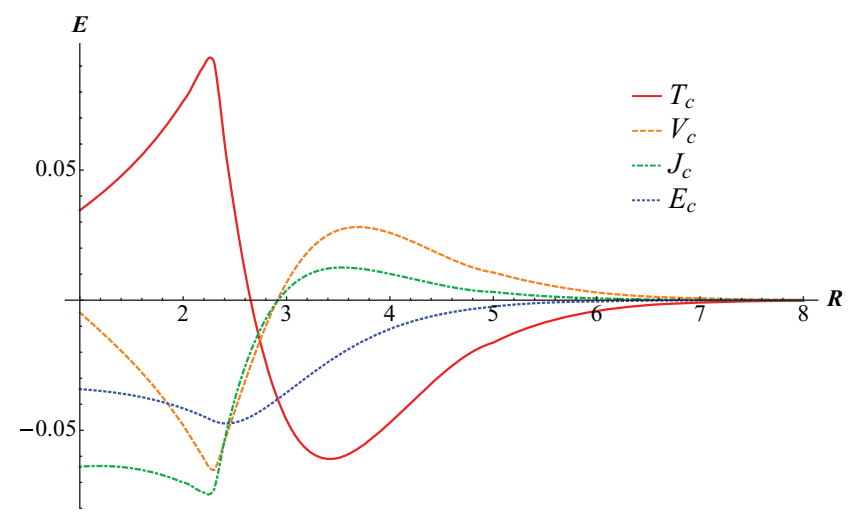

FIG. 4. UHF correlation energy components for various $R$. 
correlation stabilizes the system by reducing the kinetic energy while worsening the electrostatic interactions. This type of correlation is less familiar but is reminiscent of that observed for two electrons on a large sphere. ${ }^{27}$ UHF theory yields $\alpha$ and $\beta$ orbitals that are localized on the nuclei but it turns out that they are in fact over-localized. Although this UHF deficiency enhances electron-nuclear attraction and reduces electron-electron repulsion, it also produces an excessively high kinetic energy. Accordingly, when correlation is introduced, the electrons relax into the internuclear region where, although less comfortable Coulombically, they can move more slowly.

\section{MOMENTUM HOLES}

Before beginning our study of the momentum holes in $\mathrm{H}_{2}$, it is useful to recall what is known about the hole in the helium atom. ${ }^{16,28,29}$ We follow the analysis of Banyard and Reed, ${ }^{16}$ who showed that momentum holes in heliumlike ions are most easily understood by separating the effects of radial and angular correlation, writing

$$
\Delta M(v)=\Delta M^{\mathrm{rad}}(v)+\Delta M^{\mathrm{ang}}(v) .
$$

Radial correlation increases the probability that one electron is close to, and the other far from, the nucleus. In such arrangements, the inner electron is likely to be moving faster than the outer one and, therefore, radial correlation tends to increase $M(v)$ for moderate $v$ and decrease it for smaller $v$.

Angular correlation increases the likelihood of the electrons being on opposite sides of the nucleus, where they oscillate in phase, much as they do when confined to a large sphere. ${ }^{30}$ Because this type of oscillation tends to align the momenta of the electrons, it decreases $M(v)$ for large $v$ and increases it for moderate $v$.

The hole in the helium atom results from the superposition of radial and angular effects, and thus consists of a dip (negative region) at small $v$, a heap (positive region) at moderate $v$, and another dip at large $v$.

For stretched $\mathrm{H}_{2}$, the analogy with the helium atom breaks down because the terms "radial" and "angular" become ill-defined. However, in the spirit of Banyard and Reed, we will introduce the decomposition,

$$
\Delta M(v)=\Delta M^{\mathrm{LR}}(v)+\Delta M^{\mathrm{IO}}(v)+\Delta M^{\mathrm{pol}}(v),
$$

to isolate the left-right, in-out, and polarization components of $\Delta M(v)$. Because the only correlation introduced by FCI with the minimal STO-3G basis is the ability of the electrons to move to opposite nuclei, while the $6-311 \mathrm{G}$ basis provides additional radial freedom, and 6-311G $(d, p)$ provides correlation through polarization functions, we define these by

$$
\begin{gathered}
\Delta M^{\mathrm{LR}}(v)=\Delta M^{\mathrm{STO}-3 \mathrm{G}}(v), \\
\Delta M^{\mathrm{IO}}(v)=\Delta M^{6-311 \mathrm{G}}(v)-\Delta M^{\mathrm{STO}-3 \mathrm{G}}(v), \\
\Delta M^{\mathrm{pol}}(v)=\Delta M^{6-311 \mathrm{G}(\mathrm{d}, \mathrm{p})}(v)-\Delta M^{6-311 \mathrm{G}}(v),
\end{gathered}
$$

where each of the functions on the right-hand sides of Eq. (10) is a FCI hole in the basis set indicated.

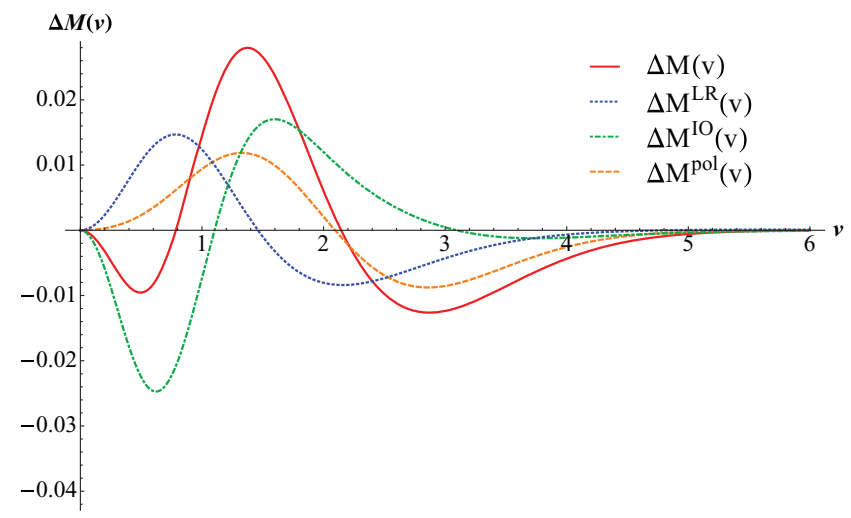

FIG. 5. HF momentum hole components for $R=1$.

Figure 5 shows the hole and its components for $R=1$. Left-right correlation favors in-phase longitudinal motion of the electrons and, therefore, increases the probability of small $v$ values. Like radial correlation in the $\mathrm{He}$ atom, in-out correlation increases the probability of large $v$ values. Polarization correlation resembles angular correlation, but the maximum and minimum of $\Delta M^{\mathrm{pol}}(v)$ occurs at larger $v$ values than those of $\Delta M^{\mathrm{LR}}(v)$. Together, these components yield a heap for $0.8<v<2.2$ and dips on either side.

Figure 6 shows the hole and its components for $R=2$. $\Delta M^{\mathrm{pol}}(v)$ is smaller than for $R=1$ but both $\Delta M^{\mathrm{IO}}(v)$ and $\Delta M^{\mathrm{LR}}(v)$ are much larger (because of the enhanced static correlation in the stretched bond $\left.{ }^{11,31}\right)$. Moreover, $\Delta M^{\mathrm{LR}}(v)$ now exhibits a second maximum at $v=3$. These effects combine to yield a deeper dip at small $v$, followed by a less pronounced heap and a shallower second dip, and then a new heap beyond $v=3$.

The second heap in $\Delta M^{\mathrm{LR}}(v)$ shows that, for $R=2$, left-right correlation favors both small $v$ and large $v$, at the expense of intermediate $v$. We surmised that the increased probability of large $v$ arises from antiphase longitudinal motion of the electrons and, to test this idea, we computed ${ }^{32}$ the momentum-dot hole $\Delta T(\tau)$, where $\tau=\mathbf{p}_{1} \cdot \mathbf{p}_{2}$. This confirms that left-right correlation increases the probability not only of in-phase motion (positive $\tau$ ) but also of antiphase motion (negative $\tau$ ).

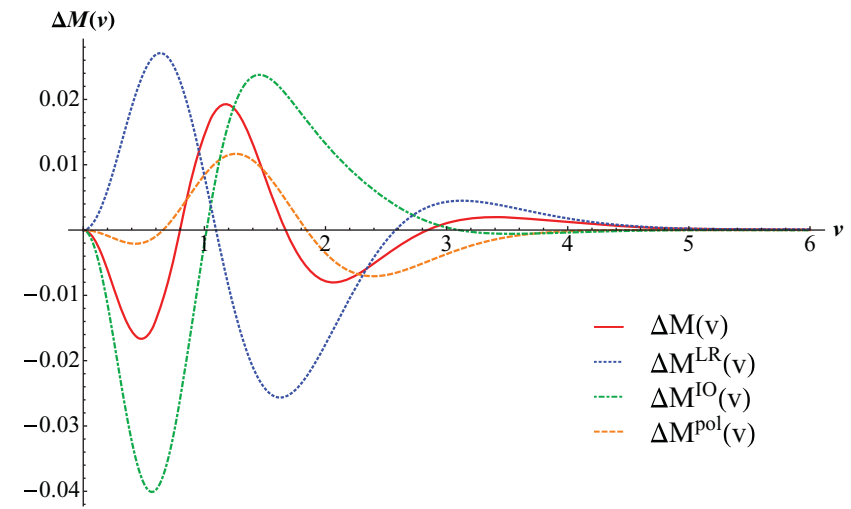

FIG. 6. HF momentum hole components for $R=2$. 


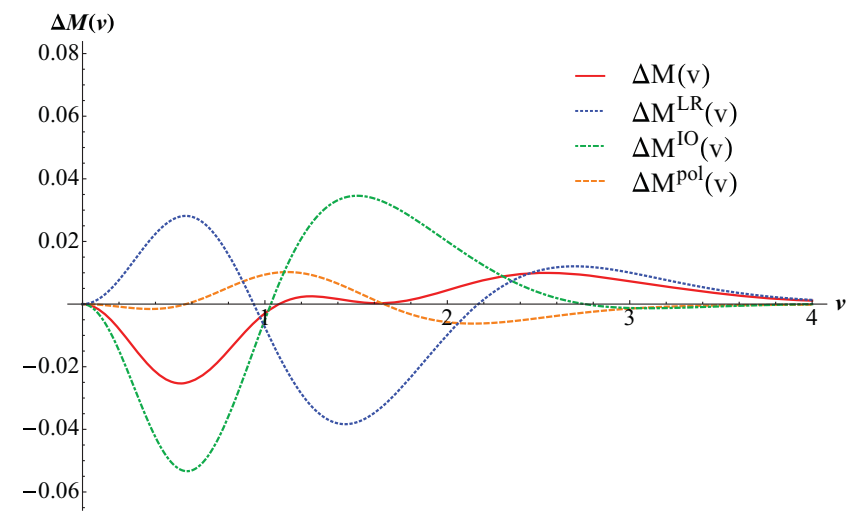

FIG. 7. RHF momentum hole components for $R=2.4$.

When the bond is stretched to $R=2.4$, which lies just beyond the triplet instability point, the RHF and UHF momentum holes become surprisingly different. In the RHF picture, the in-out effects begin to dominate and the resulting hole (Fig. 7) consists mainly of a dip at small $v$ and a heap at large $v$. In contrast, in the UHF picture, the left-right effects dominate and the resulting hole (Fig. 8) consists of a heap at small $v$, a dip at medium $v$, and a secondary heap at large $v$.

These trends persist as the bond elongates further and, for $R=10$, the RHF and UHF holes (Figs. 9 and 10) are dominated by in-out and left-right effects, respectively. (Polarization effects are negligible for such $R$.) As a result, whereas the RHF hole consists primarily of a small- $v$ dip and a large$v$ heap, perturbed by a small high-frequency ripple from the left-right effect, the UHF hole consists primarily of the leftright ripple, augmented by small contributions from the in-out effect.

In Sec. II, we discovered that the UHF position hole (Fig. 3) is negligible for $R=10$, indicating that UHF describes the relative positions of the electrons well. However, the oscillations in Fig. 10 reveal that the UHF description of the relative momenta of the electrons is fundamentally deficient. Why is this?

To identify the source of the ripples in Figs. 9 and 10, we consider a model with just the two basis functions

$$
\phi_{ \pm}(\mathbf{r})=(2 \alpha / \pi)^{3 / 4} \exp \left(-\alpha|\mathbf{r} \pm \mathbf{R} / 2|^{2}\right) .
$$

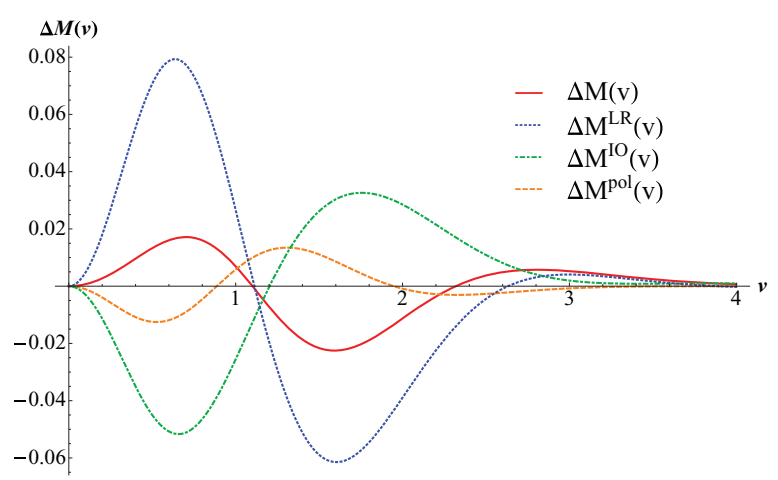

FIG. 8. UHF momentum hole components for $R=2.4$.

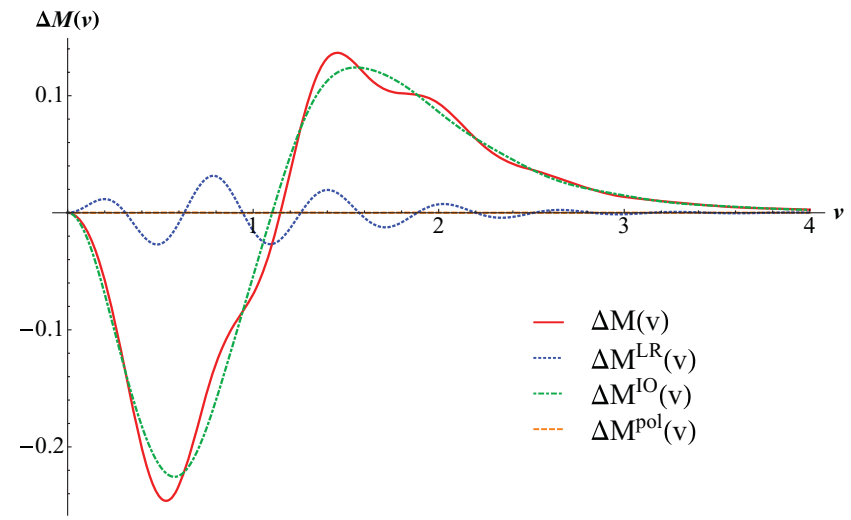

FIG. 9. RHF momentum hole components for $R=10$.

If the bond length $R$ is sufficiently large that overlap can be neglected, the UHF, RHF, and FCI wavefunctions ${ }^{33}$ in this primitive basis are

$$
\begin{gathered}
\Psi_{\mathrm{UHF}}=\left|\phi_{+}(1) \bar{\phi}_{-}(2)\right\rangle, \\
\Psi_{\mathrm{RHF}}=\left|\psi_{+}(1) \bar{\psi}_{+}(2)\right\rangle, \\
\Psi_{\mathrm{FCI}}=\left[\left|\psi_{+}(1) \bar{\psi}_{+}(2)\right\rangle-\left|\psi_{-}(1) \bar{\psi}_{-}(2)\right\rangle\right] / \sqrt{2},
\end{gathered}
$$

where the bonding and antibonding orbitals are

$$
\psi_{ \pm}=\left(\phi_{+} \pm \phi_{-}\right) / \sqrt{2}
$$

and the overbar indicates an electron of beta spin. One can then show that the momentum intracules are

$$
\begin{gathered}
M_{\mathrm{UHF}}(v)=\frac{v^{2}}{\sqrt{4 \pi \alpha^{3}}} \exp \left(-\frac{v^{2}}{4 \alpha}\right), \\
M_{\mathrm{RHF}}(v)=\frac{v^{2}}{\sqrt{4 \pi \alpha^{3}}} \exp \left(-\frac{v^{2}}{4 \alpha}\right)\left[1+j_{0}(R v) / 2\right], \\
M_{\mathrm{FCI}}(v)=\frac{v^{2}}{\sqrt{4 \pi \alpha^{3}}} \exp \left(-\frac{v^{2}}{4 \alpha}\right)\left[1+j_{0}(R v)\right],
\end{gathered}
$$

where $j_{0}(x)=\sin x / x$. Each of these contains the Maxwell distribution arising in the analogous treatment of a helium

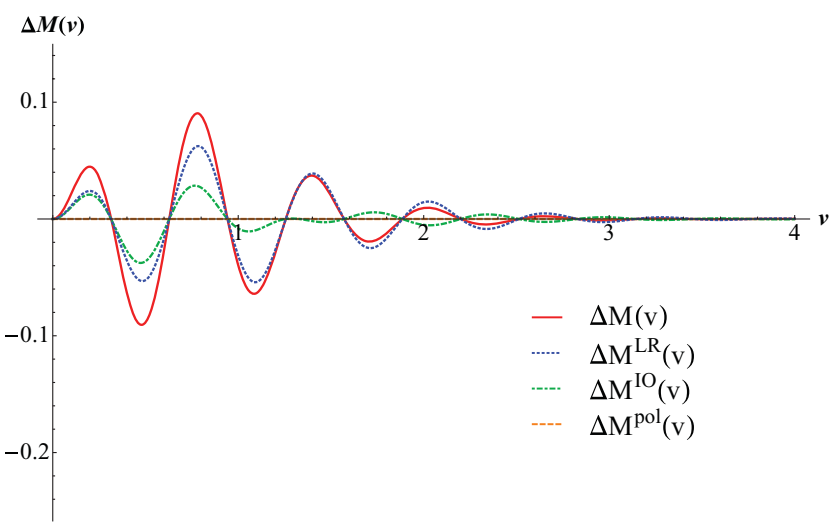

FIG. 10. UHF momentum hole components for $R=10$. 
atom. ${ }^{34}$ However, because the RHF and FCI wavefunctions are based on the two-center orbitals (13), their momentum intracules exhibit a Young-type interference pattern governed by the oscillatory factors $1+j_{0}(R v) / 2$ and $1+j_{0}(R v)$, respectively. This effect has also been seen in recent experimental measurements ${ }^{35}$ of the double photoionization of $\mathrm{H}_{2}$, where Young-type interference is observed in the correlated momentum of the ejected electrons.

The failure of the UHF intracule to capture any of the two-center interference present in the FCI intracule, therefore, explains the oscillations in the UHF hole in Fig. 10.

\section{CONCLUSIONS}

The behavior of the electrons in a bond is complicated, even in the "simple" $\mathrm{H}_{2}$ molecule, and becomes even more intricate as the bond dissociates homolytically. The RHF model is a reasonable starting point near the equilibrium bond length but, being unable to describe the breaking of an electron pair, becomes grossly inadequate as the bond is stretched. The flexibility of the UHF model allows the formerly paired electrons to move apart but can do so only by sacrificing the purity of the spin state.

In this paper, we have examined the effects of passing from the RHF or UHF models toward an exact treatment and have thereby uncovered the effects of electron correlation on electronic motion, as a function of the bond length $R$. Several observations emerge from our work:

- At all bond lengths, RHF correlation yields a conventional position hole (Fig. 2), i.e., it tends to increase the distance between the electrons. This feature of electron correlation has been familiar since the early work of Hylleraas. ${ }^{36}$ It is driven primarily by the reduction of electron-electron repulsion.

- For large $R$, UHF correlation yields an inverted position hole (Fig. 3), i.e., it tends to decrease the distance between the electrons. This feature, which is probably widespread, ${ }^{23,27}$ stems from the tendency of UHF to over-localize symmetry-broken orbitals and is driven by the reduction of kinetic energy.

- For small $R$, correlation yields momentum holes (Figs. 5 and 6) with a "dip-heap-dip" structure that results from competition between the effects of left-right correlation and in-out correlation.

- For large $R, \mathrm{RHF}$ correlation yields a large "dip-heap" momentum hole, i.e., it tends to increase the electrons' relative momentum. This is because the removal of the spurious ionic contributions in the RHF wavefunction allows the electrons to contract toward the nuclei and then to move faster.

- For large $R$, UHF correlation yields an oscillatory momentum hole, reflecting similar oscillations in the true momentum intracule. This is a quantum effect caused by Young-type interference between the two nuclear centers ("slits") and reveals that, even beyond dissociation, the momenta of the two electrons remain coupled.
We expect that these conclusions will also apply, with little modification, to other $\sigma$ and $\pi$ bonds.

\section{ACKNOWLEDGMENTS}

J. W. H. thanks the Natural Sciences and Engineering Research Council (NSERC) of Canada for funding. L.K.M. thanks the RSC for a Summer Research Scholarship. P.M.W.G. thanks the NCI National Facility for a generous grant of supercomputer time and the Australian Research Council (Grant Nos. DP0984806 and DP1094170).

${ }^{1}$ S. Goedecker and C. J. Umrigar, Phys. Rev. Lett. 81, 866 (1998).

${ }^{2}$ K. J. H. Giesbertz and E. J. Baerends, J. Chem. Phys. 132, 194108 (2010).

${ }^{3}$ M. Piris, J. M. Matxain, X. Lopez, and J. M. Ugalde, J. Chem. Phys. 133, 111101 (2010)

${ }^{4}$ P. M. W. Gill, D. L. Crittenden, D. P. O’Neill, and N. A. Besley, Phys. Chem. Chem. Phys. 8, 15 (2006).

${ }^{5}$ E. E. Dumont, D. L. Crittenden, and P. M. W. Gill, Phys. Chem. Chem. Phys. 9, 5340 (2007).

${ }^{6}$ D. L. Crittenden, E. E. Dumont, and P. M. W. Gill, J. Chem. Phys. 127, 141103 (2007).

${ }^{7}$ Y. A. Bernard, D. L. Crittenden, and P. M. W. Gill, Phys. Chem. Chem. Phys. 10, 3447 (2008).

${ }^{8}$ J. K. Pearson, D. L. Crittenden, and P. M. W. Gill, J. Chem. Phys. 130, 164110 (2009).

${ }^{9}$ J. W. Hollett and P. M. W. Gill, Phys. Chem. Chem. Phys. 13, 2972 (2011).

${ }^{10}$ A. P. Gaiduk and V. N. Staroverov, J. Chem. Phys. 133, 101104 (2010).

${ }^{11}$ V. A. Rassolov, M. A. Ratner, and J. A. Pople, J. Chem. Phys. 112, 4014 (2000).

${ }^{12}$ E. Wigner, Phys. Rev. 46, 1002 (1934).

${ }^{13}$ P.-O. Löwdin, Adv. Chem. Phys. 2, 207 (1959).

${ }^{14}$ J. A. Pople and J. S. Binkley, Mol. Phys. 29, 599 (1975).

${ }^{15}$ C. A. Coulson and A. H. Neilson, Proc. Phys. Soc. (London) 78, 831 (1961).

${ }^{16}$ K. E. Banyard and C. E. Reed, J. Phys. B 11, 2957 (1978).

${ }^{17}$ A. J. Thakkar, A. N. Tripathi, and V. H. Smith, Jr., Phys. Rev. A 29, 1108 (1984).

${ }^{18}$ B. S. Sharma and A. J. Thakkar, J. Phys. B 17, 3405 (1984).

${ }^{19}$ R. J. Boyd, C. Sarasola, and J. M. Ugalde, J. Phys. B 21, 2555 (1988).

${ }^{20}$ J. Wang, A. N. Tripathi, and V. H. Smith, Jr., J. Chem. Phys. 97, 9188 (1992).

${ }^{21}$ J. Sanders and K. E. Banyard, J. Chem. Phys. 96, 4536 (1992).

${ }^{22}$ K. E. Banyard and J. Sanders, J. Chem. Phys. 99, 5281 (1993).

${ }^{23}$ M. C. Per, S. P. Russo, and I. K. Snook, J. Chem. Phys. 130, 134103 (2009).

${ }^{24}$ A. M. Lee and P. M. W. Gill, Chem. Phys. Lett. 313, 271 (1999).

${ }^{25}$ M. W. Schmidt, K. K. Baldridge, J. A. Boatz, S. T. Elbert, M. S. Gordon, J. H. Jensen, S. Koseki, N. Matsunaga, K. A. Nguyen, S. Su, T. L. Windus, M. Dupuis, and J. A. Montgomery, Jr., J. Comput. Chem. 14, 1347 (1993).

${ }^{26}$ J. K. Pearson, P. M. W. Gill, J. M. Ugalde, and R. J. Boyd, Mol. Phys. 107, 1089 (2009).

${ }^{27}$ P. F. Loos and P. M. W. Gill, Phys. Rev. A 81, 052510 (2010).

${ }^{28}$ T. Koga, Chem. Phys. Lett. 350, 135 (2001).

${ }^{29}$ D. P. O'Neill and P. M. W. Gill, Phys. Rev. A 68, 022505 (2003).

${ }^{30}$ P. F. Loos and P. M. W. Gill, Phys. Rev. A 79, 062517 (2009).

${ }^{31}$ J. W. Hollett and P. M. W. Gill, J. Chem. Phys. 134, 114111 (2011).

${ }^{32}$ J. W. Hollett and P. M. W. Gill, (unpublished).

${ }^{33}$ A. Szabo and N. S. Ostlund, Modern Quantum Chemistry (McGraw-Hill, New York, 1989).

${ }^{34}$ P. M. W. Gill, D. P. O'Neill, and N. A. Besley, Theor. Chem. Acc. 109, 241 (2003).

${ }^{35}$ D. Akoury, K. Kreidi, T. Jahnke, T. Weber, A. Staudte, M. Schöffler, N. Neumann, J. Titze, L. P. H. Schmidt, A. Czasch, O. Jagutzki, R. A. Costa Fraga, R. E. Grisenti, R. D. Muino, N. A. Cherepkov, S. K. Semenov, P. Ranitovic, C. L. Cocke, T. Osipov, H. Adaniya, J. C. Thompson, M. H. Prior, A. Belkacem, A. L. Landers, H. Schmidt-Böcking, and R. Dörner, Science 318, 949 (2007).

${ }^{36}$ E. A. Hylleraas, Z. Phys. 65, 209 (1930). 